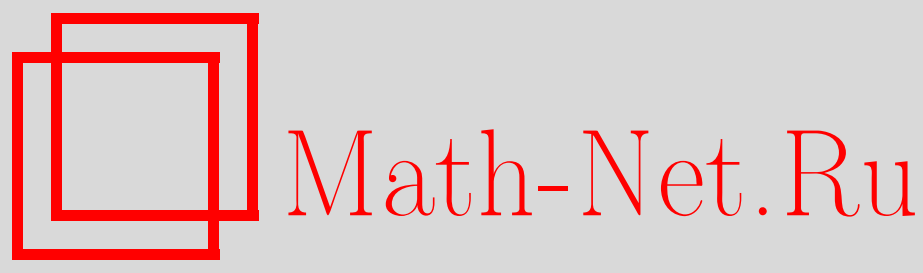

В. П. Маслов, Термодинамика флюидов. Качественное рассмотрение, ТM $\Phi, 2009$, том 161, номер 2, 224-242

DOI: https://doi.org/10.4213/tmf6434

Использование Общероссийского математического портала Math-Net.Ru подразумевает, что вы прочитали и согласны с пользовательским соглашением http: //www.mathnet.ru/rus/agreement

Параметры загрузки :

IP: 35.173 .219 .12

26 апреля 2023 г., 15:06:48

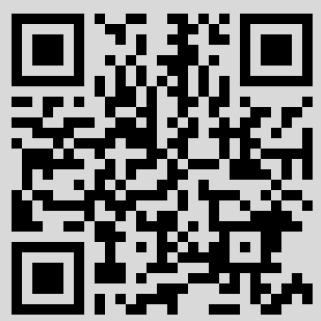




\section{ТЕРМОДИНАМИКА ФЛЮИДОВ. КАЧЕСТВЕННОЕ РАССМОТРЕНИЕ}

Рассматривается термодинамика флюидов (т.е. области газ-жидкость, в которой невозможно отличить газ от жидкости). Для этой области с помощью метода теории распределений в диофантовых уравнениях получено объяснение старых экспериментов. Ряд старых постулатов и гипотез опровергнуты, в том числе математическая аксиома о том, что распределения независимых событий перемножаются. Для слабо неидеального случая предлагается воспользоваться методом комплексного ростка, чтобы избежать применения опровергнутой ранее гипотезы Каца о сохранении хаоса.

Ключевые слова: димеры, кластеры, парастатистика, конденсат, релятивистский газ, жидкость, флюиды, термодинамика, теория распределений, теория чисел.

\section{1. ВВЕДЕНИЕ}

Шестая проблема Гильберта состоит в построении непротиворечивых аксиом в различных областях физики, в частности аксиоматики теории вероятностей и механики. Действительно, аксиоматическая теория вероятностей была построена и законы механики были аксиоматизированы. Возникла аксиоматика теории относительности. В середине XX века была построена также аксиоматика квантовой теории поля.

Если обратиться к возникновению идей этой великой теории, то стоит обратить внимание на логику диссертации одного из ее творцов - Фейнмана [1]. Исходным моментом рассуждения Фейнмана было замечательное наблюдение, которое сегодня называется фейнмановским операторным исчислением. Фейнман заметил, что если определить порядок действия некоммутирующих операторов $A$ и $B$ (т.е. оператор $A$ всегда действует первым, а $B$ - вторым) и пронумеровать их, то формула разложения в ряд Тейлора остается верной для экспоненты:

$$
e^{\stackrel{1}{A}+\stackrel{2}{B}}=\sum_{n=0}^{\infty} \frac{(\stackrel{1}{A}+\stackrel{2}{B})^{n}}{n !} .
$$

* Московский государственный университет им. М. В. Ломоносова, Москва, Россия. E-mail: v.p.maslov@mail.ru 
Это достаточно сложно проверить, поскольку

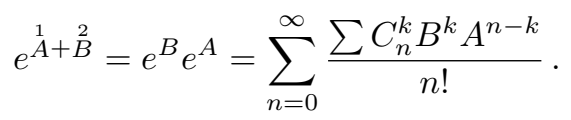

Фейнман применил эту логику нумерации некоммутирующих операторов даже для случая операторов, зависящих от непрерывного параметра, указывающего на порядок действия оператора. Отсюда возникли знаменитые фейнмановские диаграммы и фейнмановский континуальный интеграл.

Покажем логическую противоречивость (точнее, ограниченность) исходной посылки Фейнмана. Пусть $A$ и $B$ - генераторы алгебры Гейзенберга. Тогда

$$
(\stackrel{3}{A}-(A+\stackrel{4}{A} B)) f(\stackrel{1}{A}, \stackrel{2}{B}, \stackrel{3}{A}) \neq 0
$$

при $\varepsilon=0$, если [2]

$$
f(x, y, z)=\frac{e^{-i(x-z) y}-1}{x-z},
$$

что является контрпримером к общей концепции нумерации Фейнмана.

Другой пример касается логики другого великого физика - Гиббса, он также относится к эквациональной логике, основывающейся на известной аксиоме теории вероятностей о перемножении распределений независимых событий.

Предельное распределение частиц классической механики $F(p, q)(p-$ импульс, $q-$ координата), когда время $t \rightarrow \infty$, должно естественно удовлетворять стационарному уравнению Лиувилля $\{H(p, q), F(p, q)\}=0$, где $H(p, q)$ - гамильтониан. Отсюда следует, что $F(p, q)=\Phi(H(p, q))$. Если $H(p, q)=H_{1}(p, q)+H_{2}(p, q)$, причем системы, отвечающие $H_{1}(p, q)$ и $H_{2}(p, q)$, не взаимодействуют, т.е. являются независимыми, то распределения должны перемножаться: $\Phi\left\{H_{1}(p, q)+H_{2}(p, q)\right\}=\Phi\left(H_{1}(p, q)\right) \times$ $\Phi\left(H_{2}(p, q)\right)$, а это возможно только в случае

$$
\Phi(H)=e^{-c H}, \quad c=\text { const. }
$$

Гиббс в своей концепции после вывода канонического распределения получил микроканоническое распределение вида

$$
\sum_{i} N_{i}=N, \quad \sum_{i} \varepsilon_{i} N_{i}=\mathcal{E} .
$$

Чтобы согласовать эти распределения и статистику Больцмана, необходимо учитывать перестановки между частицами $N_{i}$. Значит, частицы нужно перенумеровать и все перестановки между ними считать новыми вариантами представления энергии $\mathcal{E}$. Это условие согласовывалось с аксиомой теории вероятностей, но именно оно приводило к парадоксу Гиббса.

Относительно нумерации приведу высказывание из учебника Ландау и Лифшица по квантовой механике: «В классической механике одинаковые частицы (скажем, электроны), несмотря на тождественность их физических свойств, не теряют все 
же своей "индивидуальности". Именно, можно представить себе частицы, входящие в состав данной физической системы, в некоторый момент перенумерованными и в дальнейшем следить за движением каждой из них по своей траектории; тогда в любой момент времени их можно будет идентифицировать» ([3], с. 252).

Когда математики предложили Больцману, по существу, пронумеровать частицы газа, а затем применить теорему Пуанкаре о возвращаемости или повернуть их вспять, он воскликнул: “Пойдите, поверните их!". Поэтому прежде всего мы продолжим исследование вопроса о нумерации, затронутого в работе [4].

В настоящее время получила бурное развитие так называемая молекулярная динамика. На современных компьютерах экспериментаторы изучают пронумерованные частицы, подчиняющиеся законам Ньютона, в надежде получить законы статистической физики и термодинамики. Иначе говоря, пытаются на компьютере осуществить те возражения, которые математики (Цемерло, Пуанкаре и другие) предъявляли Больцману.

Но если таких экспериментов было проведено великое множество разными экспериментаторами и начальные данные у них были различными, то общим было лишь число частиц, их суммарная энергия, а также максимальная энергия. Тогда никакая нумерация однородных частиц (шариков, дробинок, электронов) не сохраняется и их перестановка между собой может быть замечена только при возвращении к начальным условиям. Лаплас писал1): “Теория вероятностей состоит в сведении всех событий одного и того же рода к некоторому числу равновероятных случаев, то есть случаев, относительно осуществления которых мы в равной мере не осведомлены, и в определении числа тех случаев, которые благоприятны для события, вероятность которого мы ищем".

В этом случае согласно Лапласу мы пишем условия микроканонического распределения и говорим, что все варианты решения задачи равновероятны и не зависят от перестановки идентичных частиц, дробинок или шариков. Вообще, даже если эти шарики и различимы на вид, но нас интересует вопрос, сколько их штук, то с этой позиции они становятся неразличимыми, как купюры, на каждой из которых поставлен номер. И экспериментатора интересует плотность и ее распределение в зависимости, например, от высоты, температуры, давления. Даже когда мы считаем число людей в какой-либо организации, мы не различаем, кто из них начальник, а кто швейцар.

Поскольку в термодинамике мы определяем плотности, а плотности от перестановки не зависят, то возникает несогласование с аксиоматикой Гиббса. Если же от этого факта отказаться, то мы окажемся в противоречии с аксиоматикой теории вероятностей, а это означает, что распределения невзаимодействующих систем не будут перемножаться.

Однако сам основатель теории вероятностей великий Колмогоров обобщил ее, подвергнув критике, и создал теорию сложности. В этой новой аксиоматике противоречия нет, поскольку распределения независимых событий, вообще говоря, могут

1)П.-С.Лаплас. "Аналитическая теория вероятностей". Цитировано по [5], с. 307. 
не перемножаться. Понятие независимости лингвистически-философское. Например, средневековое выражение "Вассал моего вассала - не мой вассал" означает ли независимость первого от последнего? Колмогоров в аксиоматике теории вероятностей обошел эту проблему с помощью обратного определения: назовем события независимыми, если их распределения перемножаются. Это достаточное условие, но нигде не доказано, что оно необходимое. Если распределения перемножаются, то это независимые события, но не обязательно обратное. Например, в статистике Бозе-Эйнштейна или Ферми-Дирака гамильтонианы могут быть невзаимодействующими, а отвечающие им распределения не будут перемножаться. Не нужно думать, что есть перекрытие собственных функций, которое называется обменным взаимодействием. При переходе к классическому пределу $(\hbar \rightarrow 0)$ перекрытие исчезает, а симметрия относительно перестановок сохраняется.

Частицы для нас уже будут неразличимы, как купюры одного достоинства, пусть даже и пронумерованные, как в любой статистике, где мы говорим о числе объектов, поскольку перестановка объектов не меняет их числа.

Таким образом, мы приходим к новой статистике, а значит, к новой термодинамике. Это не совсем бозе-статистика, так как $N_{i} \leqslant N$, хотя $N$ и велико, это парастатистика. Мы не принимаем аксиомы бозе-статистики о том, что химический потенциал не может быть положительным, и отказываемся от аксиомы, которая носит название "закон равнораспределения", так же как и от ряда других понятий классической термодинамики, таких как идеальный газ и газ Ван-дер-Ваальса.

Соотношения микроканонического распределения не учитывают парного взаимодействия. Однако в аксиоматике новой идеальной термодинамики мы учтем вид этого взаимодействия - потенциал Леннарда-Джонса - и свойства этого потенциала.

Парная энергия в задаче рассеяния с потенциалом Леннарда-Джонса имеет вид

$$
E(\rho, r)=4 \varepsilon\left(\frac{a^{6}}{r^{6}}-\frac{a^{12}}{r^{12}}\right)\left(\frac{\rho^{2}}{r^{2}}-1\right)^{-1},
$$

где $\varepsilon$ - глубина потенциальной ямы, $a$ - эффективный радиус, $\rho$ - прицельный параметр.

Минимум $r_{1}$ и максимум $r_{2}$ при заданном $\rho$ на рис. 1 определяются из условия

$$
\frac{d E}{d r}=0
$$

В некоторой точке $\rho=\rho^{0}$ они совпадают, и, следовательно,

$$
\frac{d^{2} E}{d r^{2}}=0
$$

в некоторой точке $r^{0}$. Подсчет дает $E_{\mathrm{cr}}=0.8 \varepsilon$. Если $E>E_{\mathrm{cr}}$, то гладкого минимума на рис. 1 нет, в этом случае мы будем говорить, что "ямка" пропадает.

Этот критерий относится лишь к двум сталкивающимся частицам. Обычное рассуждение в молекулярной физике заключается в предположении симметрии среднего движения молекул по всем шести направлениям. Следовательно, друг другу 


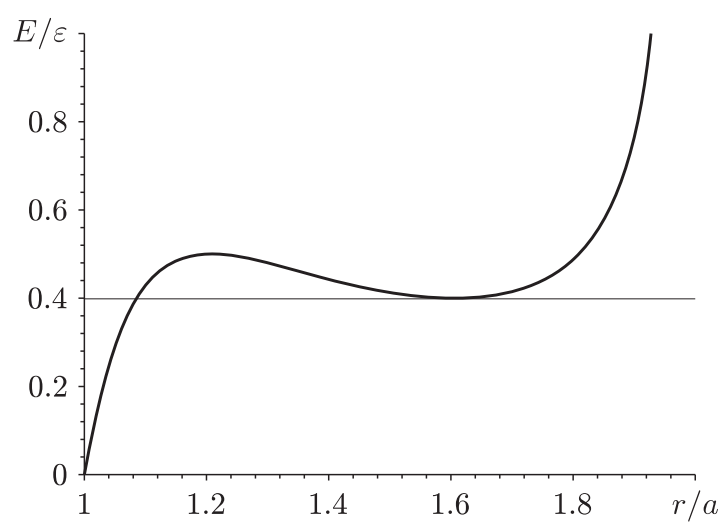

Рис. 1. Ловушка.

навстречу движется 1/12 часть всех частиц. Таких осей три, поэтому сталкивается 1/4 часть всех молекул. Их средняя энергия

$$
k T_{\mathrm{B}}=\frac{16}{5} \varepsilon,
$$

где $k$ - постоянная Больцмана. Соответствующая ей температура, по-видимому, по физическому смыслу соответствует температуре Бойля.

Как видно из рис. 1, который описывает поведение центра масс, проникновение за барьер падающих из бесконечности рассеивающихся друг на друге частиц возможно лишь в квантовом случае. Согласно теореме Понтрягина-Андронова-Витта в присутствие шума такое проникновение возможно и для классических частиц. В нашем случае по условию все варианты микроканонического распределения равновероятны. Это условие дает существенно бо́льшие амплитуды, чем в белом шуме. Поэтому можно полагать, что если число частиц $N$ превышает некоторое значение $N_{\mathrm{cr}}$, то частицы проникают через барьер при условии, что температура ниже $T_{\mathrm{B}}$, и превращаются в колеблющиеся или вращающиеся пары.

Полагая $r / a=x, \rho / a=\widetilde{\rho}$, получаем

$$
E(x)=4 \varepsilon\left(\frac{1}{x^{6}}-\frac{1}{x^{12}}\right)\left(\frac{\widetilde{\rho}^{2}}{x^{2}}-1\right)^{-1} .
$$

Производная от функции $E(x)$ равна

$$
E^{\prime}(x)=8 \varepsilon \frac{3 x^{8}-2 \rho^{2} x^{6}-6 x^{2}+5 \widetilde{\rho}^{2}}{x^{11}(-\rho+x)^{2}(x+\rho)^{2}} .
$$

После замены $y=x^{2}$ уравнение на точки возможного экстремума имеет вид

$$
3 y^{4}-2 \rho^{2} y^{3}-6 y+5 \widetilde{\rho}^{2}=0 .
$$

Отсюда

$$
\widetilde{\rho}^{2}=3 y \frac{y^{3}-2}{2 y^{3}-5},
$$




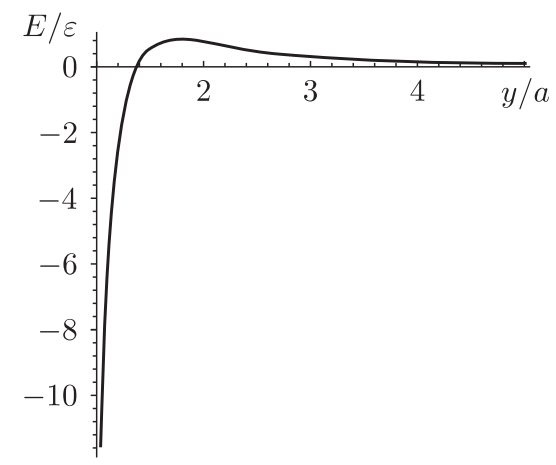

Рис. 2. Зависимость энергии $E$ от $y$.

а зависимость

$$
E(y)=4 \varepsilon\left(\frac{1}{y^{3}}-\frac{1}{y^{6}}\right)\left(\frac{\rho^{2}}{y}-1\right)^{-1}
$$

имеет вид

$$
E(y)=4 \varepsilon\left(\frac{1}{y^{3}}-\frac{1}{y^{6}}\right)\left(3 \frac{y^{3}-2}{2 y^{3}-5}-1\right)^{-1}
$$

Максимальное значение $E_{\max }=0.8$ достигается в точке $y=\sqrt[3]{5}$. Соответствующие значения $\widetilde{\rho} \geqslant 3 / \sqrt[3]{5}$.

График функции $E(y)$ (см. рис. 2) дает точки максимума при $y=y_{1}(E)<\sqrt[3]{5}$ и минимума ямы (рис. 1) при $y_{2}>\sqrt[3]{5}$.

Отметим, что прохождение классической частицы через точку $y_{2}$ не является прохождением через обычный барьер. При переходе от квантовой механики к классической эта точка не является обычной точкой поворота, и классическая частица в пределе проскальзывает за бесконечно большое время в яму.

В работе [6] мы рассматривали фокус Коровьева в варьете, когда он разбрасывает $n$ купюр над $k$ зрителями, и показали, что если число зрителей вдвое больше некоторого критического числа $k_{0}$, то с большой вероятностью половина зрителей не получит ни одной купюры. Однако если они объединятся в пары с тем, чтобы хоть кто-то из двоих получил купюры и поделил их с партнером, то с большой вероятностью все что-нибудь получат.

Оба сценария с точки зрения колмогоровской сложности равновероятны: ни одному из них нельзя отдать предпочтения. Как мы доказали, при таком каноническом распределении также имеется критическое число $N_{\mathrm{cr}}$, как в случае бозе-конденсата.

Аксиомы бозе-статистики о том, что $N-N_{\text {сr }}$ частиц переходят на нулевой уровень (останавливаются), в силу изложенного выше мы заменяем на следующую: $N-N_{\text {cr }}$ частиц образуют димеры, тримеры и другие кластеры, которые не влияют на давление, а образуют в газе броуновские частицы. 


\section{2. АБСТРАКТНАЯ ТЕРМОДИНАМИКА}

Рассмотрим абстрактное микроканоническое распределение

$$
\sum_{i=1}^{s} \varepsilon_{i} N_{i}=M, \quad \sum_{i=1}^{s} N_{i}=N,
$$

где $M, N, \varepsilon_{i}$ и $s$ - заданные числа, $N_{i}$ - целые положительные числа, $N$ - целое число. Поскольку система (8) может вообще не иметь решений, то вместо нее рассмотрим систему

$$
\sum_{i=1}^{s} \varepsilon_{i} N_{i} \leqslant M, \quad \sum_{i=1}^{s} N_{i}=N .
$$

Логарифм от числа всех возможных вариантов решения системы (9) будем называть энтропией.

Если число вариантов решений этой системы при замене правой части второго уравнения (9) на $N+1$ уменьшается, то мы говорим, что такое $N$ есть $N_{\mathrm{cr}}$. Значит при переходе от $N_{\mathrm{cr}}$ к $N_{\mathrm{cr}}+1$ в правой части второго уравнения (9) энтропия уменьшается.

Если $\varepsilon_{i}=i^{\alpha}$, где $\alpha$ - положительное число, то будем говорить, что задана абстрактная идеальная термодинамика.

Предположим, что $s$ удовлетворяет соотношению

$$
s=\frac{N(M / N)^{\gamma / \alpha}}{\alpha^{\gamma} \rho \pi^{\gamma / \alpha} \Gamma(1+\gamma / \alpha)},
$$

где $\rho=$ const - плотность в фрактальной размерности $\gamma$. Будем говорить, что $\gamma-$ размерность, $M-$ глобальная энергия.

Определим $b$ и $\kappa$ из следующих соотношений:

$$
\begin{aligned}
& \int_{0}^{\infty} \frac{p^{\alpha}}{p^{1-\gamma}}\left\{\frac{1}{e^{b\left(p^{\alpha}+\kappa\right)}-1}-\frac{N}{e^{b N\left(p^{\alpha}+\kappa\right)}-1}\right\} d p=\mathcal{E} \\
& \int_{0}^{\infty} \frac{1}{p^{1-\gamma}}\left\{\frac{1}{e^{b\left(p^{\alpha}+\kappa\right)}-1}-\frac{N}{e^{b N\left(p^{\alpha}+\kappa\right)}-1}\right\} d p=N
\end{aligned}
$$

где $b$ обратно пропорционально температуре $T$, а значение $\kappa$ пропорционально химическому потенциалу $\mu$. Соответствующие распределения были приведены в работе [7].

Теперь пересмотрим барометрическую формулу, а именно вместо

$$
\rho(z)=\rho_{0} e^{-m g z / k T}
$$

положим

$$
\rho(z)=\rho_{0} \operatorname{li}_{3 / 2}\left[-\frac{m g z+\kappa\left(\rho_{0}, T\right)}{k T}\right],
$$


где $\mathrm{li}_{3 / 2}$ - полилогарифм, а величина $\kappa\left(\rho_{0}, T\right)$, по модулю равная химическому потенциалу, удовлетворяет соотношению

$$
\int_{0}^{\infty} \frac{p^{2} d p}{\exp \left\{\frac{p^{2} /(2 m)+\kappa\left(\rho_{0}, T\right)}{k T}\right\}-1}=\rho_{0} .
$$

В экспериментах повышение давления осуществляется с помощью поршня, который можно представить как скользящую вдоль цилиндра площадку, нагруженную некоторой массой $m_{0}$. Тогда потенциал газа с нагруженной площадкой ऽ будет иметь вид $U\left(r-r_{0}\right)-m_{0} g z$, а точка равновесия, следовательно, сместится из $r_{0}=\left(x_{0}, y_{0}, z_{0}\right)$ в точку $r_{0}-m_{0} g / \frac{\partial U}{\partial z}(0)$. Следовательно, при дополнительном давлении $P^{\Delta}$ объем уменьшится на величину $V^{\Delta}=\varsigma z_{0}-m_{0} g / \frac{\partial U}{\partial z}(0)$, а так как $\left(m_{0} g\right) / \varsigma$ равно давлению $P$, то получается, что уменьшение объема пропорционально увеличению дополнительного давления $-V^{\Delta}=\delta P^{\Delta}$, где $\delta=$ const.

ЗАмечАниЕ. Таким образом, если потенциал идеального газа был $H(p)$, то мы определили гамильтониан вида $H(p, z)=H(p)-\delta_{1} P^{\Delta}$, где $\delta_{1}$ - константа, зависящая от исходного объема. Общее давление в обычной термодинамике есть сумма давления при постоянном объеме, зависящего от температуры, и дополнительного давления $P^{\Delta}$. Как известно, в изотермическом процессе в обычном идеальном газе $V \sim 1 / P$. В нашем случае это выполняется лишь для $P^{\Delta}$ и $V^{\Delta}$, где $P^{\Delta}-$ внешнее давление поршня, а $V^{\Delta}$ - уменьшение удельного объема по отношению к равновесному, возникающее при дополнительном давлении извне.

По существу, абстрактные соотношения (9) отвечают изохорическому процессу (при постоянном объеме). После небольших изменений давления немного меняется температура, затем подправляется температура, и таким образом достигается изотермичность процесса.

Введем потенциал $\Omega$, отвечающий добавленному давлению $P^{\Delta}$ и добавленному объему $V^{\Delta}$ :

$$
d \Omega=-S d T-N d \mu-P^{\Delta} d V^{\Delta} .
$$

В приведенной в работе [7] и выше парастатистике

$$
\Omega=\frac{\alpha}{\gamma} M
$$

Прежде всего уточним понятие почти идеальной жидкости. Следуя известнейшей модели гидродинамики жидкости - понятию несжимаемой жидкости с плотностью $\rho=$ const, приводящей к уравнениям Навье-Стокса, мы тем самым полагаем, что звуковые волны в жидкости отсутствуют. Мы полагаем также, что все кластеры вращаются и состоят из димеров ${ }^{2)}$.

Действительно, как известно, звуковая волна определяется как колебательный процесс "сжатие-расширение", и если $\rho=$ const, то такой процесс отсутствует. Следовательно, это означает, что совокупности молекул жидкости - димеров и других

\footnotetext{
2) Поскольку имеются кластеры с нечетным числом мономеров, то соотношение (17) может быть уточнено. Все кластеры в идеальной жидкости не осциллируют.
} 
кластеров - совершают вращательные движения и, как известно, небольшие поступательные. Эта поступательная энергия составляет приблизительно $10^{-5}$ от вращательной. Поскольку "реальная" размерность $\sigma=\gamma / \alpha$ есть отношение числа степеней свободы к степени гамильтониана как функции импульсов, то для поступательной энергии $\sigma=3 / 2$, а для вращательной $\sigma=1$. А так как поступательная энергия составляет лишь $10^{-5}$ от вращательной, то средняя реальная размерность $\sigma$ почти идеальной жидкости будет несколько превышать единицу: $\sigma=1+3 / 2 \cdot 10^{-5}$.

Следовательно, поскольку температура двухфазового состояния задана, а $N^{(1)}-$ число частиц газовой фазы, $N^{(2)}$ - число частиц жидкой фазы (димеров и кластеров), то в силу абстрактного микроканонического распределения

$$
\begin{gathered}
\sum_{i=1}^{\infty} \mathcal{E}_{i}^{(1)} N_{i}^{(1)}=M^{(1)}, \quad \sum_{i=1}^{\infty} N_{i}^{(1)}=N^{(1)}, \\
\sum_{i=1}^{\infty} \mathcal{E}_{i}^{(2)} N_{i}^{(2)}=M^{(2)}, \quad \sum_{i=1}^{\infty} N_{i}^{(2)}=N^{(2)}, \\
N^{(1)}+2 N^{(2)}=N,
\end{gathered}
$$

где $M^{(1)}=N^{(1)} / b, M^{(2)}=N^{(2)} / b$, при $\kappa \neq 0$. Мы можем определить "химические потенциалы" $\kappa^{(1)}$ и $\kappa^{(2)}$, а также число возможных вариантов разложения задачи (15) и (16).

Число решений общей задачи (15) и (16), очевидно, равно произведению числа решений задачи (15) и числа решений задачи (16). Логарифм этого произведения есть общая энтропия $S$, равная сумме $S^{(1)}+S^{(2)}$. При фиксированном $b$ условие максимальности $S$ приводит однозначно к соотношению между $N^{(1)}$ и $N^{(2)}$, а следовательно, и к соотношению между $\kappa^{(1)}$ и $\kappa^{(2)}$.

Отметим, что поскольку объем $V$ двухфазовой смеси постоянен, то давление $P$ с точностью до величины порядка $10^{-5}$ пропорционально $M^{(1)}$.

Для точного определения размерности почти идеальной жидкости можно воспользоваться формулой (10).

Принимая во внимание, что число частиц $N$ не меняется и объем общий, получим размерность $\gamma$ из соотношения

$$
\frac{(M / N)^{\gamma / 2}}{2^{\gamma} \pi^{\gamma / 2} \Gamma(1+\gamma / 2)}=\frac{M / N}{8 \pi}+\frac{10^{-5}(M / N)^{3 / 2}}{8 \pi^{3 / 2} \Gamma(1+3 / 2)} .
$$

Энтропия бозе-газа для трехмерного случая, как известно, равна

$$
S=\frac{2}{3}\left(\frac{\partial M}{\partial T}\right)_{V, \kappa} .
$$

Для размерности $\gamma / \alpha$ при фиксированных объеме и энтропии она равна соответственно

$$
S=\frac{\gamma}{\alpha}\left(\frac{\partial M}{\partial T}\right)_{V, \kappa} .
$$


Так как в нашем случае $\alpha=2$, а $\gamma>2$ вычисляется из формулы (18), то энтропия идеальной жидкости

$$
S_{\text {liquid }}=\frac{\gamma}{2}\left(\frac{\partial M^{(2)}}{\partial T}\right)_{V, \kappa_{2}} .
$$

Поэтому условие, определяющее соотношение между $\kappa^{(1)}$ и $\kappa^{(2)}$, находится из максимума $\max \left(S_{\text {liquid }}+S_{\text {gas }}\right)$ при фиксированных температуре и объеме. Тем самым определяются $N_{\text {liquid }}$ и $N_{\text {gas }}$, а также число $M^{(1)}$ газа, пропорциональное равновесному давлению смеси газа и жидкости, умноженному на общий объем $V$.

Таким образом, с помощью диофантовых уравнений теории чисел мы получаем абстрактную термодинамику без понятия давления, термодинамику изохорического процесса, когда объем спрея (смеси) постоянен. При постоянном объеме полная внутренняя энергия пропорциональна “давлению” в старом его понимании.

Поскольку мы предполагаем, что при конденсате образуются димеры, то их появление приводит, очевидно, к уменьшению энергии газа. Вычислим это уменьшение. Как известно, в задаче рассеяния имеется одна циклическая переменная $\rho_{\varphi}$, а мы как раз и предполагаем, что димеры не колеблются, а вращаются. Эта переменная одномерна, и поэтому интегрирование по ней распределения типа (11) приводит для плотности к полилогарифму $\operatorname{li}_{1 / 2}\left(e^{-\kappa}\right)$, а для энергии - к полилогарифму $\mathrm{li}_{3 / 2}\left(e^{-\kappa}\right)$.

Мы должны учесть также вклад от потенциала ямы, приведенного на рис. 1, и воспользоваться распределением, доказанным в работе [8] в случае, когда $\kappa$ есть функция от $x$. В результате получим энергию

$$
\widetilde{\mathcal{E}}=c_{2} \int_{0}^{y} \int_{0}^{\infty} \frac{\left(p_{\varphi}^{2}+u(x)\right) d p_{\varphi} d x}{e^{\left(p_{\varphi}^{2} / 2 m+u(x)+\kappa\right) / \theta}-1},
$$

где $\kappa$ находится из соотношения

$$
c_{1} \int_{0}^{y} \int_{0}^{\infty} \frac{d p_{\varphi} d x}{e^{\left(p_{\varphi}^{2} / 2 m+u(x)+\kappa\right) / \theta}-1}=\frac{N-N_{\mathrm{cr}}}{2 V},
$$

а $\theta=k T=4 E$. Это последнее равенство (обычное в молекулярной теории) следует из симметрии задачи рассеяния по всем направлениям. Здесь $c_{1}$ и $c_{2}-$ константы нормировки, которые подбираются так, чтобы при $\kappa=\infty$ температура измерялась в градусах Кельвина.

Сначала мы получаем кривые изохорического процесса, вводим внешнее дополнительное давление $\bar{P}^{\Delta}$ как внешние силы и только после этого приходим к установлению термодинамического равновесия. После того как добавляется небольшое внешнее давление, может быть определена температура Бойля.

Действительно, из химического потенциала $\kappa^{(1)}$ газа вычитается $\delta_{1} \bar{P}^{\Delta}$, поэтому

$$
P_{\text {gas }}^{0}+\bar{P}^{\Delta}=\left\{\int_{0}^{\infty} \frac{\left(p^{2} / 2 m\right) p^{2} d p}{e^{\left(p^{2} /(2 m)+\kappa^{(1)}-\delta_{1} \bar{P}^{\Delta}\right) / \theta}-1}-\widetilde{\mathcal{E}}\right\}\left(V_{\text {gas }}^{0}-\bar{V}_{\text {gas }}^{\Delta}\right)^{-1},
$$

и к правой части (24) в силу (почти) несжимаемости димеров (элементов, визуально не различимых в флюидах жидкой фазы) добавляется важный член $\bar{P}^{\Delta} V_{\text {liquid }}$,

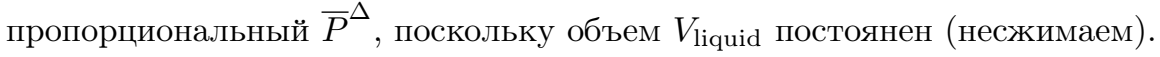




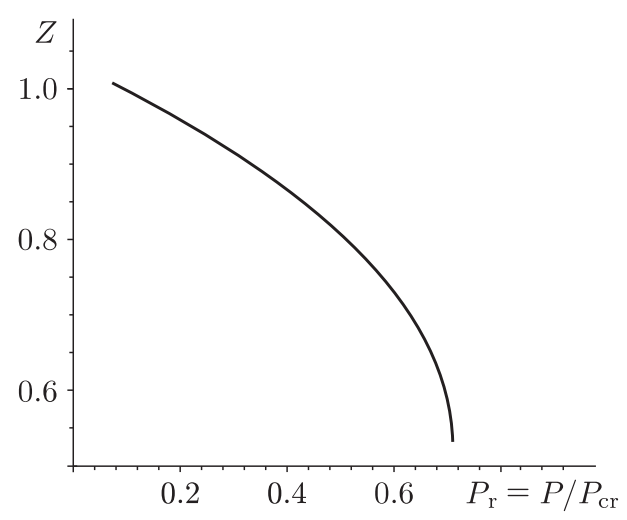

Рис. 3. Теоретическая зависимость $E\left(P / P_{\mathrm{cr}}\right)$ при $T=T_{\mathrm{cr}}-\delta$, где $\delta$ достаточно мало.

Рассмотрим теперь изотерму, т.е. добавим к постоянному $P^{0}$ принудительное (положительное и отрицательное) давление $P^{\Delta}$ за счет опускания или поднятия поршня. Тогда будет существовать температура, при которой

$$
\begin{aligned}
P_{\text {gas }}^{0}+\bar{P}^{\Delta}+P^{\Delta}= & \left(V_{\text {gas }}^{0}-V_{\text {gas }}^{\Delta}\right)^{-1}\left\{\int_{0}^{\infty} \frac{\left(p^{2} / 2 m\right) p^{2} d p}{e^{\left(p^{2} /(2 m)+\kappa^{(1)}-\delta_{1}\left(\bar{P}^{\Delta}+P^{\Delta}\right)\right) / \theta}-1}-\widetilde{\mathcal{E}}\right\}+ \\
& +\left(\bar{P}^{\Delta}+P^{\Delta} \Theta\left(P^{\Delta}\right)\right) V_{\text {liquid }},
\end{aligned}
$$

где $\Theta\left(P^{\Delta}\right)=1$ при $P^{\Delta} \geqslant 0$ и $\Theta\left(P^{\Delta}\right)=0$ при $P^{\Delta}<0$, не будет иметь минимума по $P^{\Delta}$. Естественно эту температуру положить равной $E_{\mathrm{cr}} / k$, и отсюда найти $\bar{P}^{\Delta}$. Это наиболее простой способ учета взаимодействия Леннарда-Джонса.

Уравнения среднего поля, приводящие к нелинейному интегральному уравнению первого рода [8], слишком сложны даже для подсчета поправок к химическому потенциалу. Приведенный способ, хотя он косвенный и отвечает аксиоме образования димеров, сравнительно просто определяется.

Выражение для фактора сжимаемости $Z$ при температуре ниже температуры Бойля

$$
\begin{array}{r}
Z=\frac{1}{R \theta}\left(V_{\text {gas }}^{0}-V_{\text {gas }}^{\Delta}\right)^{-1}\left\{\int_{0}^{\infty} \frac{\left(p^{2} / 2 m\right) p^{2} d p}{e^{\left(p^{2} /(2 m)+\kappa^{(1)}-\delta_{1}\left(\bar{P}^{\Delta}+P^{\Delta}\right)\right) / \theta}-1}-\widetilde{\mathcal{E}}\right\}+ \\
+\left(\bar{P}^{\Delta}+P^{\Delta} \Theta\left(P^{\Delta}\right)\right) V_{\text {liquid }}
\end{array}
$$

для изотерм при изменении $P^{\Delta}$ будет соответствовать экспериментальным графикам, подобным тем, которые изображены на рис. 1 в работе [9].

Подчеркнем, что экспериментатор не отличает газ от жидкости при $T>T_{\mathrm{cr}}$, поэтому в формулах $(25),(26)$ добавляется член, отвечающий за димеры (за жидкость).

На рис. 3 показано, как падает фактор сжимаемости при температуре, немного меньшей $T_{\text {cr }}$, при давлении, меняющемся от малого до $P_{\text {cr }}$. Далее наступает лавинообразный переход в силу того, что энергия газа при переходе через конденсат 


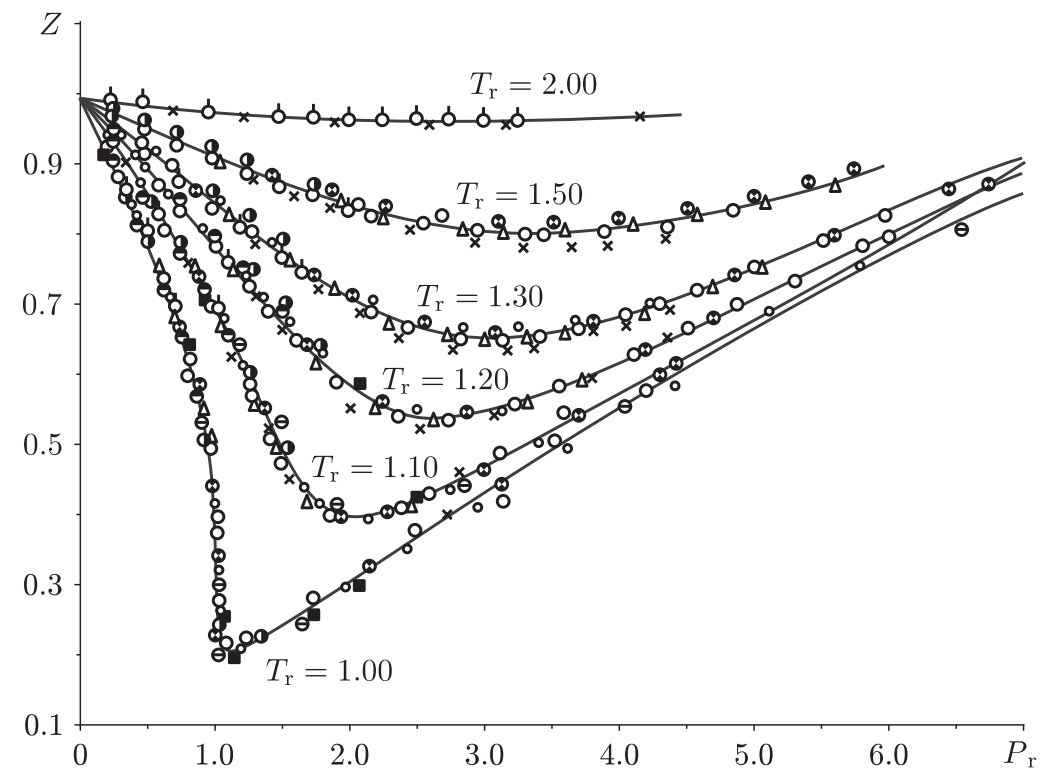

Рис. 4. Экспериментальные данные для метана (х), этилена (о), этана $(\Delta)$, пропана $(\bullet)$, н-бутана $(\bullet)$, изопентана $(\bullet)$, н-гептана $(\boldsymbol{\bullet})$, азота (১), двуокиси углерода $(\boldsymbol{\bullet})$, воды $(\mathbf{0})$ для разных значений $T_{\mathrm{r}}=T / T_{\mathrm{cr}}$. Сплошная кривая проведена согласно данным по гидрокарбону.

уменьшается за счет образования димеров и фактор сжимаемости падает до 0.2 (а не до 0.01, как в случае, если бы жидкость была полностью сжимаемой). Далее происходит почти линейный рост $Z$ в силу почти несжимаемости жидкой компоненты

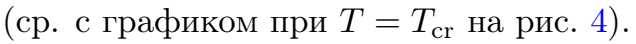

При химическом потенциале $\kappa$, достаточно большом вблизи области критического значения $E \sim 0.8 \varepsilon$, "ямку" $u(x)$ можно считать квадратичной, и переход к координатам $p_{\varphi}^{2}+d x^{2}=\xi^{2}$ при малом $d$ дает помимо увеличения $\kappa$ линейную зависимость от температуры для “вычитаемой” плотности, определяемой правой частью формулы (23). Такая замена приводит к тому, что в формуле (26) правая часть будет уменьшаться, а значит, химический потенциал будет увеличиваться. Поэтому на уровне фактора сжимаемости $Z=1$ давление газа будет меняться по параболе. В этом проявляется аналогия $\Theta=4 \cdot 0.8 \varepsilon / k$ с температурой Бойля.

Остальные графики на рис. 4 можно объяснить следующим образом. Все минимальные точки изотерм получаются для изохорического процесса. Далее, добавляя $P^{\Delta}$ или уменьшая $P^{\Delta}$, мы получаем каждую изотерму.

Отметим, что увеличивая $P^{\Delta}$, мы уменьшаем $\kappa$ и, следовательно, "выжимаем"

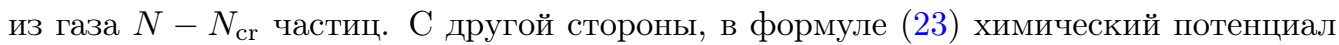
растет за счет уменьшения $y$. Поэтому при $Z=1$ именно член (22) начинает играть существенную роль в том, что давление будет меняться по параболе. Заметим, что идеальный бозе-газ, отвечающий формуле (15) для $M^{(1)}$, совпадает с обычным идеальным газом, в котором $Z=1$, при $k=\infty$, поэтому в формуле $(23) E / \varepsilon \approx 0.8$. 
Отметим, однако, что для воздуха фактор сжимаемости $Z=P V / R T$ падает до 0.02 вблизи критической точки. Вообще, для смеси газов формулы могут быть уточнены по аналогичной схеме.

Перейдем к учету парного взаимодействия. В этом случае нужно рассматривать интегральное уравнение вида

$$
W(q)=-P^{\Delta} z+\frac{\iint d p^{\prime} d q^{\prime} V\left(q, q^{\prime}\right) u\left(\left(p^{\prime}\right)^{2} / 2 m+W\left(q^{\prime}\right)\right)}{\iint d p^{\prime} d q^{\prime} u\left(\left(p^{\prime}\right)^{2} / 2 m+W\left(q^{\prime}\right)\right)},
$$

где функция $u(E)$ выбирается в виде $u(E)=\left(e^{(E-\mu) / \theta}-1\right)^{-1}, V\left(q, q^{\prime}\right)$ - эффективный потенциал Леннарда-Джонса с энергией (2). Затем следует применить метод комплексного ростка (см. п. 4.3 монографии [10]).

Отметим, что гипотеза о сохранении хаоса, сформулированная Кацем и фактически используемая во всех работах по статистической физике классических частиц, опровергнута в [11].

При уменьшении температуры число частиц и объем газа уменьшаются, а число частиц жидкости увеличивается. Так как давление определяется почти одним газом, мы вычисляем полную энергию только газа. С уменьшением температуры она уменьшается, а общее число частиц $N \sim N_{\text {liquid, }}$ поэтому фактор сжимаемости растет. При увеличении температуры минимальное значение $Z$ увеличивается. Гладкость $Z$ обеспечивается наличием небольшой сжимаемости жидкости.

Подчеркнем, что экспериментатор не отличает газ от жидкости при $T>T_{\text {cr }}$, поэтому в выражении для $Z$ (см. ниже формулу (43)) добавляется член, отвечающий за димеры (за жидкость).

Заметим, что коэффициенты диффузии, теплопроводности и вязкости вычисляются в приведенной выше термодинамике (как абстрактной, так и для флюидов) стандартным образом (см., например, [12]). Выражения для этих коэффициентов можно использовать для ламинарного течения флюидов в гидродинамике.

Однако в случае турбулентного и сверхзвукового течения ситуация существенно меняется. Для этих процессов меняются диофантовы уравнения и, следовательно, абстрактная термодинамика. Это особенно существенно для аэродинамики. Но об этом речь пойдет в следующих работах.

Теперь опишем, как будет развиваться сценарий при температуре, большей температуры Бойля, т.е. при $k T>16 \varepsilon / 5$. При этой температуре "ямка" на рис. 1 исчезает с большой вероятностью. Но с точки зрения физики очевидно, что при достаточно большом (но медленном, квазиравновесном) увеличении давления будет образовываться кристалл.

Поясним это с помощью диофантовых уравнений и закона взаимодействия Леннарда-Джонса. Дело обстоит следующим образом. В кристалле можно пронумеровать все узлы, представляющие из себя молекулы, их "перестановка" меняет кристалл. Это следует из эксперимента, когда в газ молекул одного сорта добавляется примесь молекул другого сорта. При достаточно большом давлении (или понижении температуры) эта примесь "вмораживается" в кристалл, и изменение ее положения меняет вид кристалла с разными вмороженностями. Значит, если говорить 
о молекулах одного вида, но окрашенных в другой цвет (например, об изотопах), то перестановка их номеров меняет кристалл. Это, впрочем, и так очевидно, если у кристалла есть размеры и он не превращается в тор искусственными условиями Борна-Кармана.

Разумеется, при этом потенциал Леннарда-Джонса должен быть уточнен, он будет существенно зависеть от числа частиц $N$. Эти уточнения могут быть получены с помощью теории комплексного ростка и метода вторичного квантования для классических частиц [13], [14]. В этом случае полная энергия $M^{(2)}$ должна вычисляться, как энергия статистики Больцмана [3] при том же числе частиц $N^{(2)}$, т.е. в диофантовом уравнении (16) должны учитываться все перестановки между $N_{i}^{(2)}$. Число вариантов (и энтропия) при этом существенно увеличится.

Аналогично тому, что было проделано выше, мы должны вычесть из энергии газа энергию кристаллических кластеров, образованных из конденсированных частиц (в воде они состоят приблизительно из 100 молекул из-за сложного кристалла льда, а как правило, из ближайших соседей - "гена" кристалла), а также вычесть из объема газа объем кластеров, вытеснивших газ.

Главное дополнительное соотношение, которым, в частности, нельзя пренебрегать при $k T>16 \varepsilon / 5$, - это учет отталкивания в потенциале Леннарда-Джонса для молекул газа. Для простоты мы его опустили, добавляя член $-P^{\Delta}$ непосредственно к химическому потенциалу. Это обедняло, но упрощало картину.

В задаче рассеяния потенциал взаимодействия - это эффективный потенциал вида

$$
\Phi_{\mathrm{ef}}(r)=U(r)\left(1-\frac{\rho^{2}}{r^{2}}\right)^{-1},
$$

где $U(r)$ - потенциал Леннарда-Джонса, $r=\left|q-q^{\prime}\right|$. Эта зависимость прицельного параметра $\rho$ от "точки поворота" $r_{0}$ определяется из соотношения $\Phi_{\mathrm{ef}}(r)=0$. Последнее следует также из симметрии молекулярной физики относительно направлений (см. (5)).

Далее мы предполагаем согласно термодинамической концепции, что $W(q)=\kappa$, т.е. не зависит от координат. В этом случае в уравнении (27) разные константы регуляризации могут приводить к разным значениям угла между изотермой и осью $Z=1:\left.\frac{d Z}{d P}\right|_{P=0}$ при $P=0, Z=1, T=1$. Из рис. 4 следует, что

$$
\left.\frac{d Z}{d P}\right|_{P=0, Z=1, T=1}=0.44 .
$$

В свою очередь, величина $r_{0}$ зависит от $\rho$ и $E$ в силу уравнения $(2): T=1$, $P=1,0.296 \leqslant V / R \leqslant 0.444$. То есть значение $V_{\text {cr }}$ не определено, тогда как угол (29) определен. В двойных логарифмических координатах можно определить главную степень этой зависимости, ей будет соответствовать число $\alpha$ в формулах (11) и минимальная точка изотерм на рис. 4. При дальнейшем увеличении $P^{\Delta}$ уменьшается объем газа на величину $V^{\Delta}$, но с другой стороны, уменьшается и число неконденсатных частиц. Однако поскольку энтропия при этом уменьшается, то удельный 
объем $V_{\text {gas }}^{\text {spec }}$ увеличивается и становится равным удельному объему, отвечающему этой энтропии при $\kappa>0$ в силу уравнений (11).

Рассмотрим добавку к химическому потенциалу $\kappa^{\Delta}$ за счет малой добавки к давлению $P^{\Delta}$. Добавленному давлению $P^{\Delta}$ отвечает уменьшение $V_{\text {gas }}^{\text {spec }}$ на $V^{\Delta}$. Мы получим из (27) для добавки $\kappa^{\Delta}$ (в отличие от случая $\kappa^{\Delta}=-z P^{\Delta}$; см. выше) дополнительный член, учитывающий парное взаимодействие, который выражает зависимость $V^{\Delta}$ от $P^{\Delta}$.

Теперь для идеального газа вместо соотношения, определенного в замечании, мы получаем соотношение между $P^{\Delta}$ и $V^{\Delta}$, учитывающее парное взаимодействие.

Таким образом, продолжая по мелким шагам $\Delta$, мы получим окончательный ответ в виде $T$-отображения. Подробное доказательство сходимости и применение этого понятия к уравнениям самосогласованного поля рассмотрены автором в [15].

Если чередовать увеличение давления с подправлением температуры для получения изотермы, то нетрудно убедиться, что этот процесс в силу выписанных соотношений согласуется с известным эффектом Джоуля-Томсона.

Перейдем теперь к флюидам в релятивистском газе.

Сформулируем общую теорему, отвечающую заданной гладкой функции $H(p) \geqslant 0$.

Предположим, что гладкая функция $H(p)$ при $p \rightarrow 0$ стремится к нулю не быстрее сколь угодно большой степени $p$. Пусть $E$ и $N \gg 1$ - заданные числа, $E_{1} \ll E$,

$$
E_{i}=\int_{E_{1} i}^{E_{1}(i+1)} H(p) d p^{\gamma}, \quad i=2,3 \ldots
$$

Рассмотрим соотношение

$$
\sum_{i=0}^{\infty} E_{i} N_{i}=E, \quad E_{0}=0
$$

где $N_{i}$ - целые числа, такие что $\sum_{0}^{\infty} N_{i}=N$.

Определим величину $b$ из условия

$$
\int_{0}^{\infty} \frac{H(p) d p^{\gamma}}{e^{b H(p)}-1}=E .
$$

Пусть $N_{\text {сr }}$ определяется из условия

$$
\int_{0}^{\infty}\left\{\frac{1}{e^{b H(p)}-1}-\frac{N_{\mathrm{cr}}}{e^{b H(p) N_{\mathrm{cr}}}-1}\right\} d p^{\gamma}=N_{\mathrm{cr}} .
$$

Рассмотрим уравнения для определения констант $b$ и $\kappa$ :

$$
\begin{gathered}
\int_{0}^{\infty} \frac{H(p) d p^{\gamma}}{e^{b(H(p)+\kappa)}-1}=E, \\
\int_{0}^{\infty}\left\{\frac{1}{e^{b(H(p)+\kappa)}-1}-\frac{N}{e^{b N(H(p)+\kappa)}-1}\right\} d p^{\gamma}=N .
\end{gathered}
$$


Определим величину

$$
\Omega=\int_{0}^{\infty} \ln \left\{1-e^{(-\kappa-H(p)) b}\right\} d p^{\gamma}
$$

и назовем ее термодинамическим потенциалом.

ТеоремА. А. Если $N \leqslant N_{\mathrm{cr}}$, то решение уравнений (34) существует и единственно при $\kappa \geqslant 0, b \geqslant 0$.

Б. Выражение

$$
\frac{1}{N} \sum_{i=1}^{\infty} N_{i} \varphi(H(b i))-\int_{0}^{\infty} \varphi(H(x))\left\{\frac{1}{e^{b(H(x)+\kappa)}-1}-\frac{N}{e^{b N(H(x)+\kappa)}-1}\right\} d x^{\gamma}
$$

где $\varphi(x)$ - любая ограниченная кусочно-гладкая функиия (с конечным числом разрывов), непрерывная в точках $b$, стремится по вероятности к нулю при $N \rightarrow \infty$.

В. Энтропия $S$ задачи (34) имеет вид

$$
S=\frac{\partial \Omega}{\partial b^{-1}}
$$

Г. Пусть $N>N_{c r}$. Тогда существует такое $\delta>0$, что

$$
\mathbf{P}\left\{\left|N_{0}-\left[N-N_{\mathrm{cr}}\right]\right| \geqslant \delta_{1} N_{\mathrm{cr}}\right\} \leqslant e^{-\left|N-N_{\mathrm{cr}}\right|^{\delta}},
$$

где $\delta_{1}$ - сколь угодно малое число, не зависящее от $N_{\mathrm{cr}}$, a $\mathbf{P}$ есть отношение числа вариантов, удовлетворяющих условию в фигурных скобках, к общему числу вариантов при $N=N_{\mathrm{cr}}$.

Элементы последовательности $N_{1}, N_{2}, \ldots, N_{i}, i \leqslant A / b$, где $A$ - сколь угодно большое число, не зависящее от $b$, удовлетворяют соотношению

$$
\begin{gathered}
\frac{1}{N_{\mathrm{cr}}}\left[\sum_{i=1}^{[A / b]} N_{i} \varphi(H(b i))-\int_{0}^{\infty} \varphi(H(x))\left\{\frac{1}{e^{b H(x)}-1}-\frac{N_{\mathrm{cr}}}{e^{b N_{\mathrm{cr}} H(x)}-1}\right\} d x^{\gamma}\right] \stackrel{\mathrm{P}}{\rightarrow} 0 \\
n p u \quad N_{\mathrm{cr}} \rightarrow \infty,
\end{gathered}
$$

где b определяется из соотношения (32).

Утверждения А и Б теоремы следуют из работы [16].

Далее мы будем полагать при $\gamma=3$

$$
H(p)=m c^{2} \sqrt{\frac{p^{2}}{2 m}+m c^{2}}-m^{2} c^{4},
$$

где $m$ - масса частицы, $c$ - скорость света. При добавлении константы к релятивистскому гамильтониану вида $m c^{2} \sqrt{\frac{p^{2}}{2 m}+m c^{2}}$ гамильтониан в точке $p=0$ обращается в нуль, $H(0)=0$, чтобы минимум гамильтониана отвечал нулевой энергии. 
СлЕДСТВИЕ. Предположим, что при скоростях, бо́лъших фиксированной скорости $v_{0}$, частищы релятивистского газа поглащаются стенками сосуда, являющимися черным телом лишь для высоких скоростей. Тогда средняя энергия (энергия Е, приходящаяся на одну частичу) не зависит от температуры.

Как и в работах [11], [17], рассмотрим дополнительное давление $\Delta P=P^{\Delta}$, при котором объем уменьшится на величину $\Delta V=V^{\Delta}$ пропорционально увеличению дополнительного давления $P^{\Delta}$. Получается, что уменьшение объема пропорционально увеличению дополнительного давления $-V^{\Delta}=\delta P^{\Delta}$.

Аналогично предыдущему и работе [17] вводится потенциал $\Omega(14)$ и точно так же рассматривается несжимаемая жидкость.

Интеграл по фазовому объему (30) приводит к квадратичной зависимости $E_{i}$ от $i$, а не от $i^{3 / 2}$, как для поступательной энергии [18]. Добавочная поступательная энергия порядка $10^{-5}$ добавит к абстрактной фрактальной размерности $\gamma=2$ малую добавку. В критической точке, как и для нерелятивистского случая, это даст падение фактора сжимаемости не до 0.01, как в случае $\gamma=2$, а возможно, до 0.2 .

Заметим, что для воздуха фактор сжимаемости $Z$ падает до 0.02 , а для воды до 0.25. Поскольку мы все равно не учитываем взаимодействия, которое дает вклад, подобный вкладу дополнительного положительного давления $P_{0}^{\Delta}$, то величина этой поправки несущественна. Поправку того же порядка дает учет земного притяжения. Когда процесс нуклеации и образования флюидов будет исследован в космосе, то, возможно, эти поправки и потребуют уточнения.

Таким образом, поскольку температура двухфазового состояния задана, а $N^{(1)} / V$ и $N^{(2)} / V$ - плотности частиц газовой и жидкой (димеров и кластеров) фаз, то в силу абстрактного микроканонического распределения (15)-(17) мы можем определить "химические потенциалы" $\kappa^{(1)}$ и $\kappa^{(2)}$, а также число возможных вариантов разложения задачи (15) и (16).

Энтропия релятивистского бозе-газа для трехмерного случая при фиксированных объеме и энтропии, как известно, равна

$$
\frac{S}{V}=\frac{1}{V}\left(\frac{\partial \Omega}{\partial T}\right)_{V, \kappa} .
$$

Соответственно энтропия идеальной жидкости (см. выше и [11], [17])

$$
\frac{S_{\text {liquid }}}{V}=\frac{\gamma}{2 V}\left(\frac{\partial M^{(2)}}{\partial T}\right)_{V, \kappa^{(2)}} .
$$

Поэтому условие, определяющее соотношение между $\kappa^{(1)}$ и $\kappa^{(2)}$, находится из максимума $\max \left(S_{\text {liquid }}+S_{\text {gas }}\right)$ при фиксированных температуре и объеме. Тем самым определяются величины $N_{\text {liquid }} / V$ и $N_{\text {gas }} / V$, а также $M^{(1)} / V$ газа.

Таким образом, и для релятивистского газа с помощью диофантовых уравнений теории чисел мы получаем абстрактную термодинамику без понятия давления, термодинамику изохорического процесса, когда объем спрея (смеси) постоянен. 
Как и ранее, получив кривые изохорического процесса, вводим внешнее дополнительное давление $\bar{P}^{\Delta}$ как внешние силы и только после этого приходим к установлению термодинамического равновесия. После того как добавляется небольшое внешнее давление, может быть определена температура Бойля.

Действительно, из химического потенциала $\kappa^{(1)}$ газа вычитается $\delta_{1} P^{\Delta}$, поэтому

$$
P_{\text {gas }}^{0}+\bar{P}^{\Delta}=\int_{0}^{\infty} \frac{H(p) p^{2} d p}{e^{\beta\left(H(p)+\kappa^{(1)}-\delta_{1} P^{\Delta}\right)}-1}\left(V_{\text {gas }}^{0}-\bar{V}_{\text {gas }}^{\Delta}\right)^{-1},
$$

и к этому выражению в силу (почти) несжимаемости димеров (элементов, визуально не различимых в флюидах жидкой фазы) добавляется важный член $\bar{P}^{\Delta} V_{\text {liquid }}$, пропорциональный $\bar{P}^{\Delta}$, поскольку объем $V_{\text {liquid }}$ постоянен (несжимаем).

Рассмотрим теперь изотерму, т.е. добавим к постоянному $P^{0}$ внешнее (положительное и отрицательное) давление $P^{\Delta}$, давя поршнем или поднимая его. Аналогично релятивистскому случаю найдем добавку $\bar{P}^{\Delta}$. Выражение для фактора сжимаемости $Z$ при температуре выше критической имеет вид

$$
Z=\frac{\beta}{N R}\left(V_{\text {gas }}^{0}-V_{\text {gas }}^{\Delta}\right)^{-1} \int_{0}^{\infty} \frac{H(p) p^{2} d p}{e^{\beta\left(H(p)+\kappa^{(1)}-\delta_{1}\left(\bar{P}^{\Delta}+P^{\Delta}\right)\right)}-1}+\left(\bar{P}^{\Delta}+P^{\Delta} \Theta\left(P^{\Delta}\right)\right) V_{\text {liquid }},
$$

где $\beta=1 / T$, для изотерм при изменении $P^{\Delta}$ будет соответствовать экспериментальным графикам, аналогичным изображенным на рис. 1 в работе [9]. Вблизи критической точки, по существу, релятивизм не сказывается.

Мы начали с шестой проблемы великого Гильберта. Ее в дальнейшем перефразировали в проблему аксиоматики теории вероятностей и механики. Непротиворечивую аксиоматику построили российские математики Бернштейн и Колмогоров. Эта аксиоматика содержала известное еще ранее понятие независимых событий, которое приводило к умножению вероятностных распределений. Мы видим, что эту аксиоматику, которая содержит философское понятие "независимость", необходимо изменить в самой математической теории вероятностей. В ее дискретной интерпретации, которая и является изначальной, понятие независимости нужно было изменить давно. Тогда не было бы и парадокса Гиббса. Сформулируем аксиому.

Независимыми называются такие распределения, что множество вариантов, относительно которых рассматривается вероятность события одного распределения, и множество вариантов другого распределения, когда рассматривается совместное событие, перемножаются, и, следовательно, энтропии Хартли складьваются.

Однако переход к непрерывному случаю здесь встречает затруднение, поскольку распространить меру Лебега на бесконечномерный случай невозможно, как невозможно определить меру в общем случае фейнмановского континуального интеграла. Можно определить ряд "правил игры", чтобы общаться с последним так, как если бы эта мера была.

Это значит, что язык нестандартного анализа должен быть перенесен на решетки и разработано соответствующее исчисление. В топологии эту идеологию проводит

4 Теоретическая и математическая физика, т. 161, № 2, 2009 г. 
М. Громов. Считаю, что будущее математики, физики и экономики именно за этой идеологией. Аналитическая теория чисел, комбинаторика, дискретные модели в физике, математическое квантование пространства-времени, кодирование - основы для будущего нестандартного анализа.

Благодарности. Автор благодарен А. И. Осипову и Е. Е. Тареевой за многочисленные дискуссии.

\section{Список литературы}

[1] R. Feynman, The principle of least action in quantum mechanics, Thesis Ph.D no 12-03, Prinston Univ., 1942.

[2] В. П. Маслов, Операторные методы, Наука, М., 1973.

[3] Л. Д. Ландау, Е. М. Лифшиц, Теоретическая физика. Т. 3. Квантовая механика. Нерелятивистская теория, ГИФМЛ, М., 1963.

[4] V.P. Maslov, Math. Notes, 85:5-6 (2009), 613-622.

[5] С. Г. Гиндикин, Рассказы о физиках и математиках, МЦНМО, М., 2001.

[6] В. П. Маслов, ТМФ, 159:1 (2009), 174-176.

[7] V.P. Maslov, Threshold levels in Economics, arXiv: 0903.4783.

[8] В. П. Маслов, ТМФ, 157:2 (2008), 250-272.

[9] V.P. Maslov, Russ. J. Math. Phys., 15:4 (2008), 493-510.

[10] В. П. Маслов, О. Ю. Шведов, Метод комплексного ростка, Эдиториал УРСС, М., 2000.

[11] V.P. Maslov, Math. Notes, 86:2 (2009), 293-297.

[12] И. А. Квасников, Термодинамика и статистическая физика. Т. 3. Теория равновесньх систем, Эдиториал УРСС, М., 2003.

[13] В. П. Маслов, Комплексный метод ВКБ в нелинейных уравнениях, Наука, М., 1977.

[14] А. Марадудин, Э. Монтролл, Дж. Вейсс, Динамическая теория кристаллической решетки в гармоническом приближении, Мир, М., 1965.

[15] В.П. Маслов, Комплексные марковские цепи и континуальный интеграл Фейнмана, Наука, М., 1976.

[16] А. М. Вершик, Функи. анализ и его прил., 30:2 (1992), 19-39.

[17] V.P. Maslov, Math. Notes, 86:1 (2009), 3-9.

[18] Л. Д. Ландау, Е. М. Лифшиц, Статистическая физика, Наука, М., 1964.

Поступила в редакцию 23.07.2009 